\title{
Las Pequeñas y Medianas Empresas Agroalimentarias en Venezuela y el Desarrollo Sustentable: Enfoque basado en los Principios de Manufactura Esbelta
}

\author{
Edyamira R. Cardozo ${ }^{(1)}$, Carlos Rodríguez ${ }^{(2)}$ y Wilfredo Guaita ${ }^{(1)}$ \\ (1) Universidad Nacional Experimental de Guayana, Centro de Investigaciones Gerenciales. \\ Edificio Sede de Investigación y Postgrado, Puerto Ordaz-Venezuela \\ (e-mail: edyanirac@yahoo.es; wguaita@hotmail.com) \\ (2) Universidad Politécnica de Madrid, Departamento de ingeniería de organización, \\ administración de empresas y estadísticas. Calle José Gutierrez de Abascal 2, Madrid-España \\ (e-mail: crmonroy@etsii.upm.es)
}

Recibido Nov. 08, 2010; Aceptado Dic. 27, 2010; Versión Final recibida Feb. 26, 2011

\section{Resumen}

Se analiza el proceso productivo de cuarenta y cinco pequeñas y medianas empresas productoras de queso guayanés telita ubicadas en dos municipios rurales del Estado Bolívar en Venezuela. El estudio estuvo dirigido a la identificación de los principios de manufactura esbelta y la aplicación del concepto de las Cinco "S": i) protección del ambiente; manejo de los residuos; ii) creación y mantenimiento de áreas de trabajo limpias y seguras; iii) capacitación y difusión de esta cultura; iv) procesos de autorregulación; y v) aplicación de buenas prácticas de producción alimentaria. La investigación fue de campo con alcance descriptivo y se aplicó un cuestionario a las cuarenta y cinco empresas. Los resultados demuestran que estas pequeñas y medianas empresas poseen limitaciones en sus sistemas productivos que impide su participación competitiva en el mercado.

\section{Small and Medium Size Enterprises of the Food and Agriculture Sector and Sustainable Development: Approach based on Principles of Lean Manufacturing}

\begin{abstract}
The productive processes of forty five small and medium size enterprises that manufacture guayanés telita cheese, located in two rural municipalities of the Bolivar State in Venezuela were analyzed. The objective of the study was identifying the principles of lean manufacture and the application of the concept of the Five "S": i) environmental protection; ii) waste management; iii) development and maintenance of areas of clean and safe work; iv) training and dissemination of this culture of self-regulation processes; and v) implementing best practices for food production. The study was of the descriptive type and a questionnaire was applied to the forty five companies. The results show that these small and medium size enterprises have limitations in their production systems that prevent their competitive participation in the market.
\end{abstract}

Keywords: SMEs, food production, dairy industry, lean manufacturing 


\section{INTRODUCCIÓN}

El hecho de que la economía se encuentre globalizada es, en nuestros tiempos, indiscutible, viene extendiéndose desde el mismo momento en que se iniciaron avances significativos en el campo de las comunicaciones y diversos factores han condicionado su fortalecimiento. A nivel tecnológico, los avances en el campo de la informática, las comunicaciones. A nivel económico, el crecimiento de las denominadas economías emergentes y la proliferación de los tratados de libre comercio que impulsan la competitividad en costos y precios. A nivel geopolítico, los cambios en las nuevas relaciones de poder mediante los procesos de integración de los países.

La relativa escasez de ciertos recursos naturales, han hecho que se exploren nuevas fuentes de provisión de materia prima, nuevas formas de transformación y se diversifiquen los procesos productivos que en muchos casos generan un impacto ambiental negativo. En la elaboración de los productos de la cadena alimentaria, también se ejecutan acciones que pueden afectar el ambiente, como el uso de materiales, tecnologías nocivas y la inadecuada de deposición de desechos derivados, entre otros. En este sentido, a nivel global se vienen haciendo intentos para controlar esta situación, se formulen políticas y diseñen acciones orientadas al control de residuos y emisiones que pueden afectar los ecosistemas y los niveles de sustentabilidad de los países. A este respecto, puede mencionarse el Protocolo de Kioto de 1997, que promueve las modalidades agrícolas sostenibles, y las Cumbres realizadas sobre el cambio climático.

Ahora bien, PYME'S agroalimentarias de la provincia venezolana se caracterizan por ser explotaciones familiares, con poco desarrollo organizativo y tecnológico, como también ocurre en otros países en vías de desarrollo (Zeballos, 2003; López y Castrillón, 2007). Su participación en el mercado se encuentra amenazada debido a que compiten con productos subsidiados y protegidos por el estado; por otra parte, deben garantizar la colocación de productos estandarizados, inocuos para la salud de los consumidores, lo que las obliga a incorporar elementos de mejora en sus procesos internos (Mercado, Córdova y Testa, 2007). Situación compleja, que deriva en desventajas competitivas y hace que estas unidades de producción ameriten la revisión de sus sistemas organizativos y productivos, para conducirlas a adoptar medidas de autorregulación interna, que tiendan a la eliminación de desperdicios y factores que no agreguen valor para garantizar un producto de calidad, sin arriesgar el desarrollo sustentable (Mula, Poler y García, 2005). Todo ello conlleva a la adopción del pensamiento "esbelto" y su aplicación en toda la organización.

La investigación ser realizó con el propósito de identificar los principios de manufactura esbelta en estas PYME'S, como: la aplicación de las Cinco "S", el nivel de cumplimiento de las premisas básicas para la protección del ambiente, el manejo de los residuos y la aplicación de buenas prácticas de producción alimentaria. Abarcó las unidades de producción ubicadas en los Municipios Piar y Padre Chien del Estado Bolívar, agrupadas en la Red artesanal de productores de queso guayanés telita, se aplicó un cuestionario basado en las buenas prácticas de producción alimentaria para un diagnóstico que incluyó instalaciones, servicios, características de la producción en cuanto a higiene y control, control de plagas, manejo, disposición de residuos y protección del trabajador. Los datos fueron operacionalizados a través del programa EXCEL para su tabulación, graficación y posterior análisis.

Como resultado, se encontró que las PYME'S estudiadas no están en capacidad insertarse competitivamente en los mercados internacionales y tienen limitaciones para garantizar la calidad e inocuidad del producto y no se evidenciaron acciones relacionadas al control del impacto ambiental.

\section{CONTEXTUALIZACIÓN}

La investigación se realizo en los Municipios Piar y Padre Chien del Estado Bolívar. El Municipio Piar, ubicado en el noreste del Estado, en el cruce de las coordenadas geográficas latitud norte

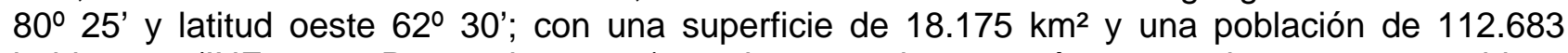
habitantes (INE, 2001-Proyeccion 2010); su importancia como área ganadera es reconocida a 
nivel nacional y local, su población pecuaria es de, aproximadamente, 60.000 cabezas de ganado vacuno de doble propósito (Acosta, 2006). El Municipio Autónomo Padre Chien, también en el noreste del estado, cuenta con una superficie de $2.275 \mathrm{Km}^{2}$, su población es de 15.000 habitantes (INE, 2008), dispersa en su territorio; es un municipio con tradición forestal y ganadera y su territorio se encuentra protegido por el Sistema de Áreas Bajo Régimen de Administración Especial (ABRAE).

Estos municipios se caracterizan por ser tradicionalmente productores de: ganado vacuno, leche, queso de cincho, mozarela y guayanés telita. Los productores se han agrupado en la Red Artesanal de Productores de Queso Guayanés Telita, ente que viene realizando estudios con apoyo de la universidad y FUNDACITE para mejorar sus procesos productivos e insertarse, competitivamente, al potencial mercado que significa la incorporación de Venezuela al MERCOSUR.

\section{El objeto de estudio:}

Constituido por la Red Artesanal de Productores de Queso Guayanés Telita, conformada por 30 queseras artesanales en el Municipio Piar y 15 en el Municipio Padre Pedro Chien. Estos productores, presentan debilidades en su infraestructura, procesos de manufactura y calificación de la mano de obra, lo cual incide notablemente en la calidad del producto, considerado, en estudios anteriores, como de alto riesgo (Rodriguez, Caldas y Ogeerally, 2009).

\section{MARCO TEÓRICO}

\section{Pequeñas y Medianas Empresas (PYME)}

La PYME es una categoría empresarial formada por un amplio y heterogéneo grupo de firmas con diferentes grados de maduración, se encuentra en todos los sectores productivos y tiene una creciente importancia en los países de la región. Diversos autores han realizado esfuerzos para clasificarla, pudiendo ser agrupadas con base en: el tipo de actividad realizada, tecnología empleada e intensidad, criterios financieros como capital invertido o volumen de ventas; o bien su capacidad empleadora. (Rosales, 2002; Guapatin, 2003; Dini y Stumpo, 2004; González, 2005)

En el estudio realizado para Observatorio de la Micro, Pequeña y mediana empresa del Banco Interamericano de Desarrollo (BID), Guapatín (2003) elabora una definición con base en los criterios que prevalecen en algunos países de la región, como son el empleo (Tabla 1), las ventas (Tabla 2) y definiciones en las que se combinan el sector y su capacidad empleadora (Tabla 3).

Tabla 1. Definiciones con base en el empleo

\begin{tabular}{|l|c|c|c|}
\hline País Definición & Micro & Pyme & Grande \\
\hline Argentina & 1 a 5 & 6 a 200 & Desde 201 \\
\hline Brasil & 1 a 9 & 10 a 99 & Desde 100 \\
\hline Colombia & 1 a 9 & 10 a 200 & Desde 201 \\
\hline Costa Rica & 1 a 4 & 5 a 70 & Desde 70 \\
\hline Guatemala & 1 a 10 & 11 a 50 & n.d. \\
\hline Honduras & 1 a 10 & 11 a 100 & Desde 101 \\
\hline Rep. Dominicana & 1 a 10 & \multicolumn{2}{|c|}{ n.d. } \\
\hline Venezuela & 1 a 5 & \multicolumn{4}{|c|}{} \\
\hline
\end{tabular}


Tabla 2. Definiciones con base las ventas. (Millones de dólares)

\begin{tabular}{|c|c|c|c|}
\hline País Definición & Micro & Pyme & Grande \\
\hline Chile & 0 a 60.000 & 60.000 a 2.500 .000 & Desde 2.500 .000 \\
\hline Panamá & 0 a 150.000 & 150.000 a 2.500 .000 & Desde 2.500 .000 \\
\hline
\end{tabular}

Tabla 3. Definiciones con base en la capacidad empleadora y el sector

\begin{tabular}{|c|c|c|c|c|}
\hline País & Sector & Micro & Pyme & Grande \\
\hline México & Manufactura & 30 & 31 a 500 & + de 501 \\
\hline & Comercio & 5 & $6-100$ & + de 101 \\
\hline & Servicios & 20 & $21-100$ & + de 101 \\
\hline
\end{tabular}

Como puede observarse, a nivel de la región no hay un criterio pre-establecido para caracterizar este tipo de empresas; lo que si esta demostrado, es su impacto en el empleo y su capacidad de ubicarse en los diversos sectores productivos.

En Venezuela, el Decreto Ley 1547 (Decreto con Fuerza de Ley para la Promoción y Desarrollo de la Pequeña y Mediana Industria) considera la PYME como un ente productivo, "generador de empleos e inversiones" que tiene su peso específico como "dinamizador de la economía del país"; en un intento de establecer los criterios para su caracterización y consiguiente regulación señala que este sector no cuenta con "una Ley que la regule, situación que ha traído como consecuencia el surgimiento de diversas disposiciones con el fin de regular su desarrollo, algunas de ellas poco aplicables a la situación del mismo."

Este mismo instrumento legal, en su Artículo 3, aborda la definición "unidad de explotación económica realizada por una persona jurídica que efectúe actividades de producción de bienes industriales y de servicios conexos". La clasificación atiende a los criterios capacidad empleadora y volumen de ventas, a saber:

Pequeña Industria: Entre once (11) y cincuenta (50) trabajadores y ventas anuales entre nueve mil una (9.001) Unidades Tributarias (UT) y cien mil (100.000) UT.

Mediana Industria: Entre cincuenta y un (51) y cien (100) trabajadores y ventas anuales entre cien mil una (100.001) UT y doscientos cincuenta mil (250.000) UT.

Dado que la PYME se encuentra en todos los sectores de la producción, en importante utilizar el término "empresa" y no "industria", pues esta última acepción incorpora restricciones conceptuales que limitan su definición, clasificación y homogenización de los datos para su análisis.

A los efectos del estudio, se considera pequeña y mediana empresa (PYME), la definición de Cardozo (2007) quien señala que son "unidades económicas que efectúan actividades de producción de bienes y servicios; que mantengan una fuerza laboral de entre once (11) y cien (100) trabajadores directos; con activos entre 50.000 a 500.000 UT y ventas anuales entre 100.000 y 250.000 UT."

La PYME se encuentra en todas los sectores de la producción y se define como agroalimentaria por dedicarse específicamente a la elaboración de productos para el consumo humano o animal y en el caso que ocupa la investigación la materia prima es de origen animal (ganadería). Para las PYME'S agroalimentarias objeto de este estudio, es muy importante lograr la tecnificación y estandarización de sus procesos, pues corren el riesgo de desaparecer del mercado por no cumplir con la normativa sanitaria y comercial existente (López y Castrillón, 2007). 


\section{Queso Guayanés Telita}

En Venezuela, el circuito lácteo se compone de tres grandes rubros: leche líquida, en polvo y la industria quesera. Esta última, absorbe el 55\% de la producción de leche (Boscan y Sandrea, 2004). Ahora bien, los quesos pueden clasificarse por sus características internas, externas, por su forma de elaboración considerandos, tipo de coagulación, grado de maduración o bien por su textura. En un sistema de clasificación más sencilla, se dividen en quesos frescos, madurados (blandos, semi-duros, duros, azules) y fundidos (Ercolini, D. et. al., 2004).

Dentro de los quesos frescos, los más importantes son el queso tipo mozzarella, de mano, guayanés, y telita (Maldonado, Llanca y Oyón, 2007; Colina y Xiquez, 2007; Lima y Massino, 2008). El queso blanco venezolano tipo guayanés telita, es un queso de pasta cocida e hilada, único de Venezuela, elaborado exclusivamente con leche cruda de vaca, con una presentación típica y completamente artesanal, muy consumido en el país; es un producto de sabor suave, aspecto lechoso, bajo en sal, medianamente graso y de textura blanda. (Márquez y García, 2007; Rodríguez, Caldas y Ogeerally, 2009).

Según la norma venezolana COVENIN 3822-2003 (2003), el queso "telita" es un queso sin madurar listo para el consumo poco después de su fabricación. Se caracteriza por ser una masa firme, homogénea y sin ojos, que es el resultado de un proceso de amasado y estirado en caliente luego de la coagulación de la leche. Debe su denominación a la región Guayana, zona de origen y líder de producción del mismo. De hecho, se considera como un producto típico de la región.

\section{Manufactura esbelta}

La manufactura esbelta tiene su génesis en la empresa automotriz japonesa y en la última década del siglo pasado se acuña como término por investigadores del Massachusetts Instituto of Technology asignados al proyecto -Intemational Motor Vehicle Program- (IMVP). Se sustenta en la implementación de estrategias producción, de calidad y mejora continua. Un proceso de manufactura es "esbelto" cuando logra el desarrollo de sus productos optimizando sus insumos, mano obra, espacios, inversión y tiempo obteniendo mayor satisfacción de los clientes internos y usuarios. Un sistema en el cual se produce con menos esfuerzo de la gente, menos espacio, menos capital, menos tiempo y además sus productos poseen menos defectos. (Cruz, 2004; Pires y Machado, 2006; Ballesteros Silva, 2008 y Padilla 2010).

Son atributos de estos sistemas de producción, entre otros: productos de forma, dimensiones y acabado requeridos, uso de técnicas de justo a tiempo para eliminar desperdicios, creación de sistemas que ayudan a los empleados a producir sin defectos, tener relaciones con los proveedores para compartir las necesidades de ambos, eliminar actividades que no agregan valor (Báez, Limón, Tiapa y Rodríguez. 2010). Ello implica una producción sustentada en la mejora continua y en actividades estimulantes y retadoras para los colaboradores.

\section{Desarrollo sustentable}

Es la noción de desarrollo que asocia al crecimiento de los pueblos, los aspectos eco-ambientales para garantizar la sostenibilidad de las futuras generaciones. Fernández B. (2004), señala que el proceso de la sustentabilidad, requiere de una agenda que incluya "todas las propuestas normativas que tienen que ver con las políticas públicas, trátense de economía, medioambiente, urbanismo, sanidad o educación." Esta afirmación implica considerar:

Lo ecológico: que permita preservar la diversidad de ecosistemas y las especies en un equilibrio dinámico. Lo económico: permitiendo la generación de riqueza en forma y cantidades adecuadas logrando su redistribución, el fomento de un intercambio equitativo en la sociedad y la diversificación de la capacidad productiva para el fortalecimiento de la actividad económica (producción y consumo). 
Lo social: mediante la adopción de valores que generen el comportamiento armónico del hombre con la naturaleza que lo rodea; así como el mantenimiento de un adecuado nivel de vida que incluya empleo, educación y seguridad, promoviendo la solidaridad y potenciando la organización de la sociedad civil y la participación.

Lo político: con el desarrollo de estructuras democráticas, el empoderamiento de la población la reducción de la dependencia del estado y el fomento de relaciones de solidaridad entre comunidades y regiones. Entre sus características se encuentran: Su apreciación en el tiempo, pues viene del pasado, está en el presente y forma parte del futuro; disminución de la dependencia de la comunidad en relación al ambiente que le es propio y sus condiciones naturales; su intangibilidad, pues aún cuando este allí, se vive y se experimenta como algo natural y no se evidencia hasta que se tiene real conciencia de él y por eso se le "ve" como algo de nuevo que no existió antes; es impulsado por de actividades estimulantes, capaces de superar el fenómeno del estancamiento; y, esta caracterizado por un conjunto de fases sucesivas irreversibles, que van de lo simple a lo complejo; o sea de estadio inferior a uno superior.

Para que las PYME'S productoras de queso guayanés telita se encuentren insertas en este concepto de desarrollo, requieren de un proceso estandarizado de producción con la implementación normas asociadas a las buenas prácticas de fabricación (BPF) incluyendo el control de los residuos que puedan afectar el ambiente; garantizar, asimismo, la inocuidad del producto y evitar enfermedades de transmisión alimentaria (ETA). Todo lo anterior conlleva a la reconsideración de su proceso productivo con innovaciones conducentes a la optimización en el uso de los recursos, la eliminación de los factores que no agreguen valor y el consecuente ajuste de su estructura de costos.

\section{METODOLOGÍA}

La investigación fue de campo con alcance descriptivo. La población estuvo conformada por 45 pequeñas y medianas empresas agrupadas en la Red Artesanal de Productores de Queso Guayanés Telita y la muestra final estuvo conformada por 30 unidades productivas. El instrumento utilizado fue un cuestionario basado en las buenas prácticas de producción alimentaria en el cual se incluyeron aspectos como: instalaciones, servicios, características de la producción en cuanto a higiene y control, control de plagas, manejo y disposición de residuos. Para la operacionalización de los datos, se utilizó la herramienta EXCEL, que permitió su análisis, graficación y posterior interpretación.

\section{RESULTADOS}

Características de las PYME'S estudiadas: contienen rasgos de explotación familiar. Su capacidad empleadora es de 139 trabajadores directos y 452 indirectos y su producción diaria es $6.246 \mathrm{Kg}$. del producto. Internamente poseen un alto sentido de pertenencia y mantienen una relación de permanencia con sus proveedores de leche. Su proceso productivo se representa en la figura 1.

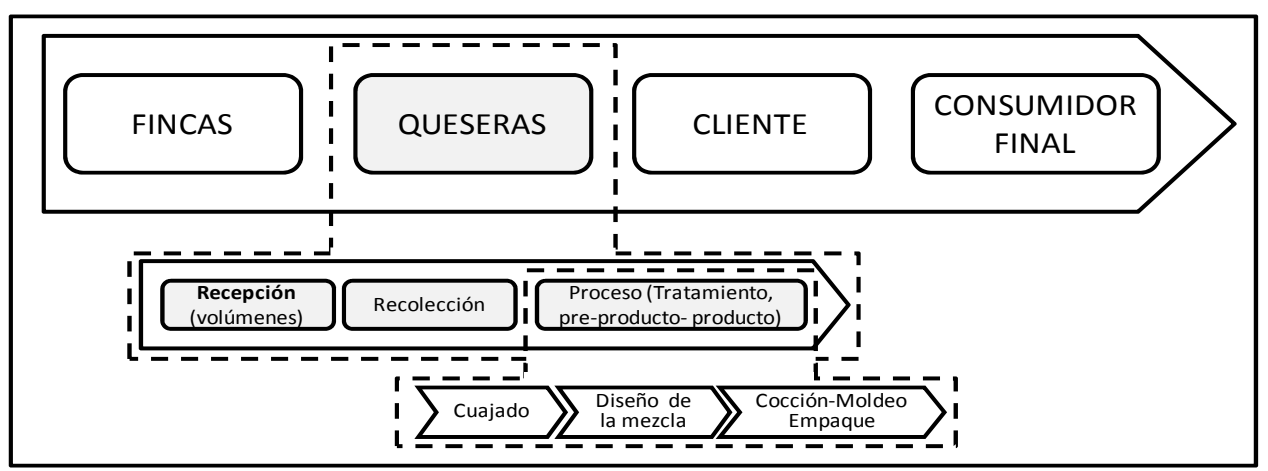

Fig. 1: Situación actual PIME’S Queseras. Cardozo, 2010 (2) 
Aplicación de principios vinculados a la manufactura esbelta: No se observaron factores vinculados con los principios de producción esbelta, son básicamente productoras artesanales, que, en algunos casos, cuentan con controles de calidad referidos a acidez de la mezcla, temperatura, textura y densidad de la masa. No fueron encontrados los aspectos: mantenimiento y organización de áreas de trabajo, capacitación, difusión de la cultura de producción esbelta y procesos de autorregulación.

Protección del ambiente: En este aspecto se consideró el sistema de recolección de la materia prima, hallándose que un 93\%, no lo posee, tal como lo refleja la figura № 2 .

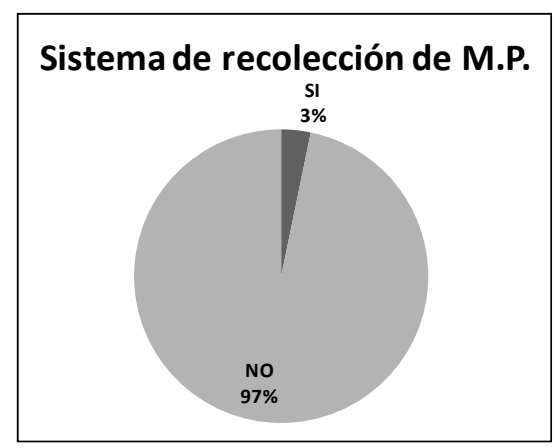

Fig. 2: Sistema de recolección de la Materia Prima.

Los productores, en su mayoría, utilizan recipientes de plástico, los cuales pueden constituirse elementos nocivos para la salud si no se aplica un adecuado sistema de limpieza de los mismos. Dado que el queso telita se produce a partir de leche cruda, se infiere que este es un aspecto que presenta una oportunidad de mejora. En cuanto a la utilización del agua, el $52 \%$ no cuenta son un sistema de aducción de agua potable ni de aguas servidas. Ver figura № 3.

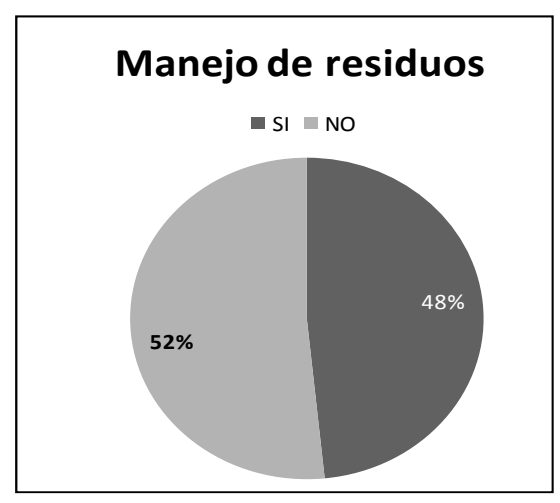

Fig. 3: Manejo de residuos.

El manejo de los residuos, incluyendo el suero lácteo, genera pasivos ambientales debido a que los mismos van al ambiente sin ningún tipo de control, generando polución de insectos y distintos tipos de plagas (moscas, mosquitos, entre otros), pueden afectar también los afluentes de agua de la zona.

Aplicación de buenas prácticas de producción alimentaria. Este aspecto fue asociado a la existencia de la permisología para funcionar y el sistema de protección del trabajador. En las figuras 4 y 5 los resultados. 


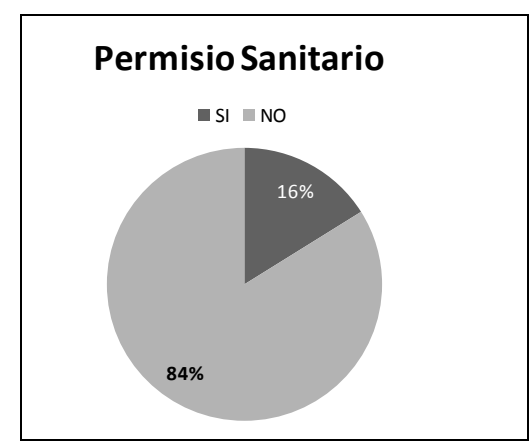

Fig. 4: Permiso Sanitario.

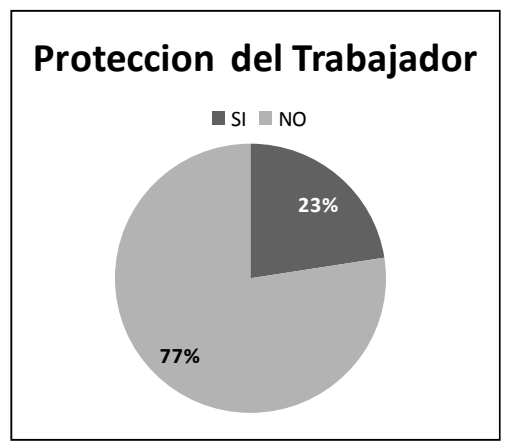

Fig. 5: Protección del trabajador.

De los productores consultados, el $16 \%$ posee permiso sanitario y por ende cumple con las buenas prácticas producción alimentaria. Es de hacer que notar sólo el 8\% del total, aplica cuatro controles asociados a la calidad, referidos a acidez de la mezcla, temperatura, textura y densidad de la masa.

El $77 \%$ no cuenta con sistemas de protección al trabajador, como uniformes, botas, gorros, guantes. El trabajador realiza las tareas prácticamente desprotegido y sin equipo de protección personal por lo que se encuentra expuesto a las condiciones del ambiente en el cual se ejecutan y están sometidos a riesgos de enfermedades propias de la labor, se destaca asimismo que los operarios no poseen Certificado de Salud, requerido en el caso de este tipo de las empresas.

\section{CONCLUSIONES}

Las PYME'S agroalimentarias estudiadas evidencian un proceso productivo tipo artesanal, con una ordenamiento horizontal, propio de estas explotaciones familiares. Los factores vinculados con los principios de producción esbelta no fueron evidenciados, por lo que existen oportunidades de mejora en estos aspectos.

En necesario que en estas PYME'S se implementen los controles de calidad en todas las áreas, para garantizar la calidad del producto y la seguridad en su consumo. De igual forma, que atiendan las políticas y estrategias de protección del ambiente.

En estas condiciones las empresas estudiadas no están en capacidad insertarse competitivamente en los mercados internacionales e inclusive existe riesgo en la continuidad de su permanencia en el mercado nacional.

Una forma de hacerlas más competitivas es incorporarlas progresivamente en el contexto de los sistemas de manufactura esbelta. La propuesta que se haga, además de integrar los aspectos característicos de este tipo de explotación, deberán incluir: el diseño organizacional, la filosofía de gestión, el diseño de cargos, las prácticas operativas, las estrategias de control de calidad; así como las herramientas propias de los sistemas de manufactura esbelta. Dadas sus características de pequeñas empresas de producción artesanal, pueden aplicarse, dentro de las herramientas esbelta: $5 \mathrm{~S}$, kanban, jidoka, poka joke y justo a tiempo.

\section{REFERENCIAS}

Acosta, R., Uso de los recursos de las microrregiones para el desarrollo de la ganadería de carne en Venezuela. X Seminario de Pastos y Forrajes 2006. (en línea), http://avpa.ula.vel congresos/seminario_pasto_X/Conferencias/A6-Rafael\%20Acosta.pdf. Acceso: 18 de Enero (2011).

Báez, Y.; Limón, J.; Tlapa, D. y Rodríguez, M., Aplicación de Seis Sigma y los Métodos Taguchi para el Incremento de la Resistencia a la Prueba de Jalón de un Diodo Emisor de Luz. Información Tecnológica. ISSN 0718-0764. (en línea), 21(1), (2010).

http://www.scielo.cl/pdf/infotec/v21n1/art11.pdf. Acceso: 23 de Febrero (2011). 
Ballesteros, S.P., Algunas reflexiones para aplicar la manufactura esbelta en empresas colombianas. Revista Scientia Et Technica. 14(1), 223-228 (2008).

Boscán, M. y Sandrea, M., Análisis de los componentes del circuito lácteo venezolano. Revista de Ciencias Sociales (RCS) X (1), 131 - 147 (2004).

Cardozo, E., La conceptualización de microempresa, microemprendimientos y unidad productiva de pequeña escala. Revista Copérnico Año IV (6), 23-30 (2007).

Colina, P. y Xiquez A. La producción artesanal de queso guayanés en los municipios Piar y Padre Chien, Estado Bolívar. Revista Autana. Departamento Hombre y Ambiente. Universidad Nacional Experimental de Guayana. 1, 54-60 (2007).

Cruz, V. A. Sistema de Gestión de la Calidad en el Apoyo a la Implementación de Estrategias de Producción Ajustada. Información tecnológica. ISSN 0718-0764. (en línea), 15(6), 2004. http://www.scielo.cl/scielo.php?pid=S0718-07642004000600010\&script=sci_arttext Acceso: 23 de Febrero (2011).

COVENIN 3822-2003. Norma venezolana - Queso de pasta hilada. Publicaciones FONDONORMA, 4, (2003).

Dini, M. y Stumpo, G., Programas de fomento de la articulación productiva. Pequeñas y medianas empresas y eficiencia colectiva. Estudios de casos en America Latina. Compilación para CEPAL. Siglo XXI Editores, s.a. de cv. Buenos Aires, Argentina (2004).

Ercolini, D. y otros 5 autores, PCR-DGGE fingerprints of microbial succession during a manufacture of traditional water buffalo mozzarella cheese. Journal of Applied Microbiology. ISSN 1365-2672. (en línea) 96(2), 2004. http://onlinelibrary.wiley.com/doi/10.1046/j.13652672.2003.02146.x/abstract. Acceso: 15 de Febrero (2011)

Fernández Buey, Francisco. Cuidar la Tierra: Filosofía y práctica de la sostenibilidad, 2004. http://www.ecoportal.net/content/view/full/25876. Acceso: 17 de Julio (2010)

González, T., Problemas de la definición de la microempresa. Revista Venezolana de Gerencia. Universidad del Zulia, ISSN 1315-9984, (en línea) 10(031), 2005. http://redalyc.uaemex.mx/redalyc/src/inicio/ArtPdfRed.jsp?iCve=29003104. Acceso: 25 de Enero (2011)

Guaipatín, C., Observatorio MIPYME: Compilación estadística para 12 países de la Región. (2003) http://www.cipi.gob.mx/Biblioteca_Digital_CIPI/bibliografiabasica/Observatorios_PYME/62659 observatoriomipyme.pdf. Acceso: 15 de Ägosto (2010)

INE, Instituto Nacional de Estadística. Censo 2001. http://www.ine.gov.ve/seccion/poblacion /magnitudestructura/Trabajo.asp?CodigoEstado=07\%20\&TipoPublicacion=Proyecciones\&AreaDe Publicacion=poblacion \&AnoBaseCenso $=2001 \&$ CodigoCuadro=Cuadro_06\&ControlHref $=7 \&$ strHref =MunicipioPiar\&strMunicipioX=Municipio\$Piar Acceso: Febrero, 15 (2011).

Lima, J. y Massino, S., Manual de Detección de Fallas de una Línea Piloto de Producción de Quesos basado en Conocimiento Experto. Información Tecnológica. ISSN 0718-0764, (en línea) 19(3), 2008. http://www.scielo.cl/scielo.php?script=sci_pdf\&pid=S0718-07642008000300010 \&lng=es\&nrm=iso\&tlng=es. Acceso: 15 de Febrero (2011).

López, F. y Castrillon, P., Teoría económica y algunas experiencias latinoamericanas relativas a la agroindustria. Libros EUMED (en línea) www.eumed.net/libros/b2007/304, 2007. Acceso: 20 de Febrero (2011). 
Maldonado, R., Llanca L. y Oyón R., Volúmenes de leche de vaca destinados a la producción del queso de mano en el Estado Aragua-Venezuela. Revista Agroalimentaria. ISSN 1316-0354 (en línea) 13(26), 2008. http://www.scielo.org.ve/scielo.php?pid=S131603542008000100010\&script=sci_arttext. Acceso: 20 de Febrero (2011)

Marquez, J.G, y García, C.E., Efecto de la nisina sobre la microflora patógena del queso blanco artesanal tipo"telita" elaborado en una quesera de Upata, Estado Bolívar, Venezuela. Revista de la Sociedad Venezolana de Microbiología. ISSN 1315-2556. (en línea) 27(2). 2007. http://www.scielo.org.ve/scielo.php?script=sci_arttext\&pid=S1315-25562007000200010\&lng= en\&nrm=iso\&ignore=.html. Acceso: 21 de Febrero (2011)

Mercado, A.; Córdova, K. y Testa, P.,. Tendencias organizativas y tecnológicas de la industria agroalimentaria global y su manifestación en Venezuela. Agroalimentaria ISSN 1316-0354 (en línea) 12(24), 2007,http://www.scielo.org.ve/scielo.php?pid=S1316-03542007000100007 \&script=sci_arttext. Acceso: 23 de Febrero (2011)

Mula, J.; Poler, R. y García, J.P., Evaluación de Sistemas para la Planificación y Control de la Producción. Información Tecnológica. ISSN 0718-0764 (en línea) 17(1), 2006. http://www.scielo.cl/scielo.php?pid=s0718-07642006000100004\&script=sci_arttext. Acceso: 24 de Febrero (2011)

Padilla, L., Lean manufacturing-Manufactura esbelta/ágil. Universidad Rafael Landívar Facultad de Ingeniería. Revista Ingeniería Primero, ISSN: 2076-3166 (en línea) 15, 2010. http://www.tec.url.edu.gt/boletin/URL_15_MEC01.pdf. Acceso: 23 de Febrero (2011).

Pires, A.M; Machado, V.C. (2006) Gestión por Procesos en el Diseño de las Organizaciones. Revista Información Tecnológica. ISSN 0718-0764 (en línea) 2006, 17(1), http://www.scielo.cl/scielo.php?pid=S0718-07642006000100005\&script=sci_arttext. Acceso: 22 de Febrero (2011).

Rodríguez, C.; Caldas, L. y Ogeerally, P., Calidad sanitaria en queso artesanal tipo "telita". Upata, Estado Bolívar, Venezuela. Revista de la Sociedad Venezolana de Microbiología. ISSN 1317-973X (en línea), 2009, 29(2), http://redalyc.uaemex.mx/redalyc/pdf/1994/199 414957006.pdf. Acceso: 25 de Enero (2011).

Rosales, R., Estrategias gerenciales para la pequeña y mediana empresa. Ediciones IESA, Caracas. (2002).

Zeballos, E., Micro, pequeñas y medianas empresas en América Latina, (2003), http://www.eclac.org/publicaciones/xml/6/19286/Zevallos.pdf. Acceso: 24 de Febrero (2011). 